\title{
TWO-PHASE FLUID SIMULATION USING A DIFFUSE INTERFACE MODEL WITH PENG-ROBINSON EQUATION OF STATE*
}

\author{
ZHONGHUA $\mathrm{QIAO}^{\dagger}$ AND SHUYU SUN $\ddagger$
}

\begin{abstract}
In this paper, two-phase fluid systems are simulated using a diffusive interface model with the Peng-Robinson equation of state (EOS), a widely used realistic EOS for hydrocarbon fluid in the petroleum industry. We first utilize the gradient theory of thermodynamics and variational calculus to derive a generalized chemical equilibrium equation, which is mathematically a secondorder elliptic partial differential equation (PDE) in molar density with a strongly nonlinear source term. To solve this PDE, we convert it to a time-dependent parabolic PDE with the main interest in its final steady state solution. A Lagrange multiplier is used to enforce mass conservation. The parabolic PDE is then solved by mixed finite element methods with a semi-implicit time marching scheme. Convex splitting of the energy functional is proposed to construct this time marching scheme, where the volume exclusion effect of an EOS is treated implicitly while the pairwise attraction effect of EOS is calculated explicitly. This scheme is proved to be unconditionally energy stable. Our proposed algorithm is able to solve successfully the spatially heterogeneous two-phase systems with the Peng-Robinson EOS in multiple spatial dimensions, the first time in the literature. Numerical examples are provided with realistic hydrocarbon components to illustrate the theory. Furthermore, our computational results are compared with laboratory experimental data and verified with the Young-Laplace equation with good agreement. This work sets the stage for a broad extension of efficient convex-splitting semi-implicit schemes for numerical simulation of phase field models with a realistic EOS in complex geometries of multiple spatial dimensions.
\end{abstract}

Key words. surface tension, convex splitting, Peng-Robinson equation of state, diffusive interface models, gradient theory

AMS subject classifications. 65N30, 65N50, 49S05

DOI. $10.1137 / 130933745$

1. Introduction. Modeling and simulation of multiphase fluid systems have been a major effort in reservoir engineering $[8,15,29,30]$ as well as in environmental protection [5, 28], such as subsurface CO2 sequestration [19]. Understanding liquid droplets, gas bubbles, phase interfaces, and capillary pressure relies on calculating and simulating accurately the physics of multiphase fluid systems, especially capturing the phenomena occurring within the interface between phases.

Understanding and modeling the interface between phases have been approached by at least three methodologies. The first methodology is to model it at the molecular scale using either molecular dynamics simulation or molecular Monte Carlo simulation $[4,9]$, with a certain intermolecular potential function (e.g., Lennard-Jones potential) being assumed. For example, a recent study has been carried out to predict $\mathrm{CO} 2-$ water interfacial tension under pressure and temperature conditions of geologic

*Submitted to the journal's Computational Methods in Science and Engineering section August 20, 2013; accepted for publication (in revised form) April 15, 2014; published electronically August 12,2014 .

http://www.siam.org/journals/sisc/36-4/93374.html

$\dagger$ Department of Applied Mathematics, The Hong Kong Polytechnic University, Hung Hom, Hong Kong (zhonghua.qiao@polyu.edu.hk). This author's work was partially supported by Hong Kong Research Council GRF grant PolyU 2021/12P and NSFC/RGC Joint Research Scheme N_HKBU204/12.

${ }^{\ddagger}$ Corresponding author. Division of Physical Sciences and Engineering (PSE), King Abdullah University of Science and Technology (KAUST), Thuwal 23955-6900, Kingdom of Saudi Arabia (shuyu. sun@kaust.edu.sa). This author's work was supported in part by the project entitled "Simulation of Subsurface Geochemical Transport and Carbon Sequestration," funded by the GRP-AEA Program at King Abdullah University of Science and Technology (KAUST).

B708 


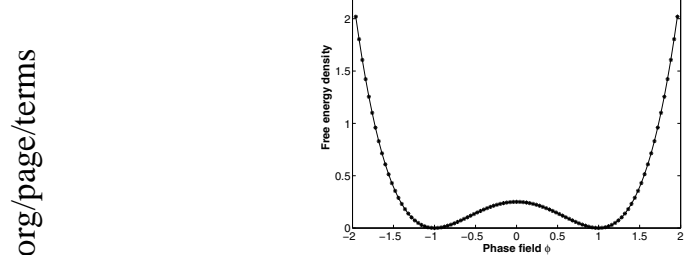

(a)

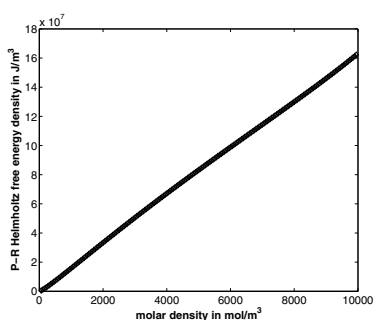

(b)

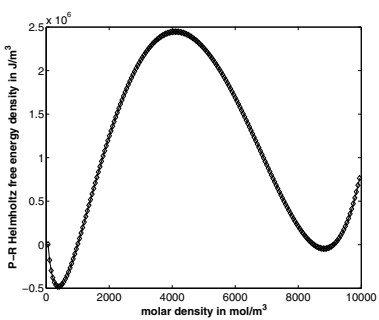

(c)

FIG. 1. Free energies of a simplified double-well model and the Peng-Robinson model.

CO2 storage using molecular dynamics simulations [20]. In the second methodology, known as diffuse interface theory [21], gradient theory [2, 14], or phase field theory [1], one describes the interface as a continuum three-dimensional entity separating the two bulk single-phase fluid regions. In this approach, molar or mass density is constant in either of the two neighboring bulk regions, but it changes continuously within the interface. The third approach, known as sharp interface modeling, is to use a zero-thickness two-dimensional entity to model the interface $[13,24]$, where the molar density experiences a jump across the interface. This approach cannot provide details within the interface itself, but it can be used to predict the shape and the dynamics of the interface once the interface tension is given. Modeling approaches combining different scales have also been used. For example, the diffusive interface model and molecular simulation have been coupled to study CO2 hydrate formation [31].

In this paper, we focus on the diffuse interface modeling of multiphase fluid systems. Diffuse interface models have been used to predict quantitatively the surface tensions of chemicals $[2,14,17,18]$ and to simulate qualitatively the immiscible multiphase flow in a channel [1]. In the former application, only one-dimensional spatial dimension has been considered. In the latter application, a simple double-well potential is usually used (thus simulation being only qualitative) rather than a more realistic equation of state (e.g., the Peng-Robinson equation of state [22]), which is more accurate but computationally more challenging. It is not possible to set quantitatively meaningful parameters of a simple double-well potential to simulate realistic hydrocarbon species in an oil-gas two-phase system. To illustrate the difference between the simple double-well model and a more realistic model (e.g., Peng-Robinson) as well as to demonstrate the computational challenges of the latter, we plot and compare the free energy as a function of the phase field (in the simple double-well model) or molar density (in the Peng-Robinson model), as in Figures 1(a) and 1(b), respectively. Here Figure $1(\mathrm{~b})$ is the result of isobutane $\left(n \mathrm{C}_{4}\right)$ at $350 \mathrm{~K}$. The nonconvexity of the double-well model is clear, while the free energy of the Peng-Robinson model is approximately linear. Only after being subtracted by a linear function does the nonconvexity of the Peng-Robinson model become visually noticeable (shown in Figure 1(c)). It is precisely this tiny nonconvex perturbation from the linear trend that causes phase separation, and thus needs to be captured accurately, which gives one computational challenge. Another computational challenge of the Peng-Robinson model is that the molar densities of the two energy-lowest positions (which correspond to gas and liquid states) differ by orders of magnitudes. The Peng-Robinson equation of state is the most popular equation of state used in oil reservoir simulation. To the best of our knowledge, simulations on diffuse interface models with the Peng- 
Robinson equation of state using efficient and accurate algorithms have not been conducted in two- or three-dimensional spaces. This challenge probably arises from the fact that certain tricky computational issues need to be circumvented for numerical treatment of diffuse interface models with the Peng-Robinson equation of state, as will be discussed in this paper.

The main purpose of this study is to provide efficient numerical methods for simulating a diffuse interface model with the Peng-Robinson equation of state for twophase fluid systems. To study the static behavior of such a system, we will solve a second-order parabolic equation, which is from the gradient flow of the corresponding total Helmholtz free energy. A Lagrange multiplier is used to enforce mass conservation. The fourth-order approach is a conserved phase-field formulation. It is a better approach if the dynamics of the two-phase fluid system is considered since it carries more physics (correct diffusion). However, our focus here is to obtain static solution of such a system, so that both the second-order approach with a Lagrange multiplier and the fourth-order approach are well applicable. Numerically, the second-order formulation is easier to implement than the fourth-order formulation. Numerical stability demands very small time steps for conventional time marching schemes. To overcome this serious difficulty, we propose an energy stable scheme with convex splitting of the energy functional. Recently, energy stable schemes have attracted considerable attention for the study of gradient flow systems, where the energy stability is guaranteed regardless of the time step size, in the sense that the energy is monotonically nonincreasing in discrete time. A convex splitting of the energy functional method was exploited by Eyre [7], to study the unconditionally energy stable time discretization of the Cahn-Hilliard equation, where the energy functional was decomposed into convex and concave parts, with the convex part treated implicitly and the concave part explicitly in the time discretization. Eyre's idea was generalized to a variety of gradient flow systems and has gained much success [3, 10, 11, 12, 25, 32, 33, 34, 35]. Energy stable schemes from other approaches can be found in $[6,23,26,36,37,38]$ and the references therein.

The rest of this paper is organized as follows. In section 2 , we will state the mathematical modeling equations. We will show how the minimization of Helmholtz free energy will be reduced to a diffusion partial differential equation (PDE) with a nonlinear source term (known as the Euler-Lagrange equation corresponding to the minimization problem). In section 3, we convert the time-independent PDE into a transient PDE as a solution strategy. An unconditionally energy stable time marching scheme together with a mixed finite element space discretization is developed to solve the transient PDE. Our algorithm maintains mass conservation, and it also guarantees the decrease of total Helmholtz free energy with time steps. In section 4, the proposed numerical scheme is applied to a case study of isobutane $\left(n \mathrm{C}_{4}\right)$ at various temperatures. Finally, concluding remarks are provided in section 5. Among other conclusions, we will highlight the consistency of our simulation with the well-known Young-Laplace equation.

\section{Mathematical model of fluid systems with diffuse interface.}

2.1. Formulation by minimizing total Helmholtz free energy. We consider a fluid system consisting of a fixed number of species on a fixed domain with a spatially uniform-distributed given temperature. In this work, we are interested in the equilibrium state of the system. According to the second law of thermodynamics, we know its total Helmholtz free energy achieves a global minimum at the equilibrium state. We first describe the computation of such total Helmholtz free energy $F$. 
We denote by $M$ the number of components in the fluid mixture and by $n_{i}$ the molar concentration of the component $i$. We let

$$
\mathbf{n}=\left(n_{1}, n_{2}, \ldots, n_{M}\right)^{T}=\frac{\left(N_{1}, N_{2}, \ldots, N_{M}\right)^{T}}{V}
$$

be the molar concentrations of all components and $n=n_{1}+n_{2}+\cdots+n_{M}$ be the molar density of the fluid. According to the gradient theory, one of the most popular thermodynamic theories for inhomogeneous fluids, the total Helmholtz energy density, has two contributions, one (i.e., $F_{0}(\mathbf{n})$ ) from the thermodynamic theory of homogeneous fluids and the other one (i.e., $F_{\nabla}(\mathbf{n})$ ) from inhomogeneity of the fluid. That is,

$$
\begin{aligned}
F(\mathbf{n}) & =F(\mathbf{n} ; T, \Omega)=\int_{\Omega} f(\mathbf{n} ; T) d \mathbf{x} \\
& =F_{0}(\mathbf{n} ; T, \Omega)+F_{\nabla}(\mathbf{n} ; T, \Omega) \\
& =\int_{\Omega} f_{0}(\mathbf{n} ; T) d \mathbf{x}+\int_{\Omega} f_{\nabla}(\mathbf{n} ; T) d \mathbf{x} .
\end{aligned}
$$

Here $f_{0}(\mathbf{n})$ is the contribution of Helmholtz free energy density (i.e., Helmholtz free energy per unit volume of the fluid) from the homogeneous fluid theory, and $F_{\nabla}(\mathbf{n})$ is the contribution of Helmholtz free energy density from the concentration gradient. The homogeneous term $f_{0}(\mathbf{n})=f_{0}(\mathbf{n} ; T)$ can be computed by using the equation of state (EOS) approach. We will describe a specific form of an EOS, called the PengRobinson EOS, in section 2.3, which is the most popular EOS for modeling petroleum fluids consisting of oil and gas. The inhomogeneous term or the gradient contribution $f_{\nabla}(\mathbf{n})$ can be modeled by a simple quadratic relation:

$$
f_{\nabla}(\mathbf{n})=\frac{1}{2} \sum_{i, j=1}^{M} c_{i j} \nabla n_{i} \cdot \nabla n_{j},
$$

where $c_{i j}$ is the influence parameter. The parameter $c_{i j}$ is a function of temperature and molar concentrations, but it depends on molar concentrations only weakly. Consequently, for a given fixed temperature $T$, it is often justified to assume that $c_{i j}$ is a constant. We will also review a model for computing $c_{i j}$ in section 2.4.

Let $H$ be a space of functions with certain regularity (for example, we may identify $H$ as the Hilbert space $\left.\left(H^{1}(\Omega)\right)^{M}\right)$. Since the molar concentration $\mathbf{n}$ at equilibrium minimizes the total Helmholtz free energy $F$, the mathematical statement of the problem is formulated as follows: find $\mathbf{n} \in H$ satisfying

$$
F(\mathbf{n})=\min _{\widetilde{\mathbf{n}} \in H} F(\widetilde{\mathbf{n}})
$$

subject to

$$
\int_{\Omega} \mathbf{n} d \mathbf{x}=\mathbf{N}
$$

Here $\mathbf{N}=\left(N_{1}, N_{2}, \ldots, N_{M}\right)^{T}$ is a given constant vector representing the fixed amount of material for each component in the system. Thermodynamic theory suggests that the total Helmholtz free energy $F$ achieves its minimum at the equilibrium state but only for a closed system with fixed volume and temperature. The condition that the volume is fixed corresponds to the domain $\Omega$ here being fixed. The condition that the system be closed corresponds to the material conservation of each component in the domain, i.e., (2.3). 
2.2. Formulation of equilibrating chemical potential. It is well known from variational principles [27] and from thermodynamics [8] that at equilibrium, the total chemical potential $\mu$ must equilibrate spatially for each component. That is,

$$
\mu_{i}(\mathbf{x})=\text { constant } \forall \mathbf{x} \in \Omega, i=1,2, \ldots, M .
$$

The total chemical potential has two contributions, one from homogeneous fluid theory and one from the gradient theory:

$$
\mu_{i}=\mu_{0, i}+\mu_{\nabla, i} .
$$

The homogeneous fluid contribution of chemical potential is straightforward to compute:

$$
\mu_{0, i}=\left(\frac{\partial F_{0}}{\partial N_{i}}\right)_{T, V, N_{1}, \ldots, N_{c-1}, N_{c+1}, \ldots, N_{M}}=\left(\frac{\partial f_{0}}{\partial n_{i}}\right)_{T, n_{1}, \ldots, n_{c-1}, n_{c+1}, \ldots, n_{M}} .
$$

The gradient contribution of chemical potential can be derived using variational calculus:

$$
\mu_{\nabla, i}=\frac{1}{2} \sum_{k, j=1}^{N} \frac{\partial c_{k j}}{\partial n_{i}} \nabla n_{k} \cdot \nabla n_{j}-\sum_{j=1}^{M} \nabla \cdot\left(c_{i j} \nabla n_{j}\right) .
$$

If we assume that the influence parameter $c_{i j}$ is independent of the molar concentration $\mathbf{n}$, this equation reduces to

$$
\mu_{\nabla, i}=-\sum_{j=1}^{M} c_{i j} \nabla^{2} n_{j} .
$$

With this assumption, the chemical equilibrium condition (2.4) becomes

$$
-\sum_{j=1}^{M} c_{i j} \nabla^{2} n_{j}=\mu_{i}-\mu_{0, i}(\mathbf{n}), \quad i=1,2, \ldots, M,
$$

where $\mu_{i}$ is the total chemical potential of component $i$, which is a constant. This chemical equilibrium equation (2.5) is a system of coupled nonlinear second-order elliptic PDEs. We note that (2.5) can be derived from minimizing (2.2) with constraint (2.3) by using the method of Lagrange multipliers and variational calculus. In this work, we shall develop an efficient numerical algorithm to solve this PDE system for single-component two-phase fluids.

2.3. Peng-Robinson EOS for modeling bulk properties. The PengRobinson EOS [22] is the most popular model for computing the fluid equilibrium property of petroleum fluids in reservoir engineering and oil industries. For completeness, we briefly describe this EOS here. With this model, the Helmholtz free energy $f_{0}(\mathbf{n})$ of a homogeneous fluid is given by

$$
\begin{gathered}
f_{0}(\mathbf{n})=f_{0}^{\text {ideal }}(\mathbf{n})+f_{0}^{\text {excess }}(\mathbf{n}), \\
f_{0}^{\text {ideal }}(\mathbf{n})=R T \sum_{i=1}^{M} n_{i}\left(\ln n_{i}-1\right),
\end{gathered}
$$

Copyright (C) by SIAM. Unauthorized reproduction of this article is prohibited. 


$$
f_{0}^{\text {excess }}(\mathbf{n})=-n R T \ln (1-b n)+\frac{a(T) n}{2 \sqrt{2} b} \ln \left(\frac{1+(1-\sqrt{2}) b n}{1+(1+\sqrt{2}) b n}\right) .
$$

Here $T$ is the temperature of the mixture and $R$ is the universal gas constant (approximately $8.31432 \mathrm{~J} \mathrm{~K}^{-1} \mathrm{~mol}^{-1}$ ). Two parameters, i.e., the energy parameter $a=a(T)$, and the co-volume parameter $b$, are utilized in the Peng-Robinson EOS.

This EOS model relies on the procedure to compute its two parameters $a$ and $b$. For a mixture, these parameters are related to those of the pure fluids by mixing rules:

$$
a(T)=\sum_{i=1}^{M} \sum_{j=1}^{M} y_{i} y_{j}\left(a_{i} a_{j}\right)^{1 / 2}\left(1-k_{i j}\right), \quad b=\sum_{i=1}^{M} y_{i} b_{i},
$$

where $a_{i}$ and $b_{i}$ are the Peng-Robinson parameters for pure-substance component $i$, and $y_{i}=n_{i} / n$ is the mole fraction of component $i$. The binary interaction coefficient $k_{i j}$ of the Peng-Robinson EOS is usually computed from experimental correlation, and $k_{i j}$ is assumed to be constant for a fixed species pair.

The pure-substance Peng-Robinson parameters $a_{i}$ and $b_{i}$ are computed from the critical properties of the species

$$
\begin{aligned}
& a_{i}=a_{i}(T)=0.45724 \frac{R^{2} T_{c_{i}}^{2}}{P_{c_{i}}}\left(1+m_{i}\left(1-\sqrt{\frac{T}{T_{c_{i}}}}\right)\right)^{2}, \\
& b_{i}=0.07780 \frac{R T_{c_{i}}}{P_{c_{i}}} .
\end{aligned}
$$

The critical temperature $T_{c_{i}}$ and critical pressure $P_{c_{i}}$ of a pure substance are intrinsic properties of the species, and they are available for most substances encountered in engineering practice. The above formula for $a_{i}$ contains a parameter $m_{i}$ for modeling the influence of temperature on $a_{i}$. The parameter $m_{i}$ is experimentally correlated to the acentric parameter $\omega_{i}$ of the species by the following equations:

$$
\begin{aligned}
& m_{i}=0.37464+1.54226 \omega_{i}-0.26992 \omega_{i}^{2}, \quad \omega_{i} \leq 0.49, \\
& m_{i}=0.379642+1.485030 \omega_{i}-0.164423 \omega_{i}^{2}+0.016666 \omega_{i}^{3}, \quad \omega_{i}>0.49 .
\end{aligned}
$$

The acentric parameter can in turn be computed by using critical temperature $T_{c_{i}}$, critical pressure $P_{c_{i}}$, and the normal boiling point $T_{b_{i}}$ :

$$
\omega=\frac{3}{7}\left(\frac{\log _{10}\left(\frac{P_{c_{i}}}{14.695 \mathrm{PSI}}\right)}{\frac{T_{c_{i}}}{T_{b_{i}}}-1}\right)-1=\frac{3}{7}\left(\frac{\log _{10}\left(\frac{P_{c_{i}}}{1 \mathrm{~atm}}\right)}{\frac{T_{c_{i}}}{T_{b_{i}}}-1}\right)-1 .
$$

From the thermodynamic theory, the pressure of homogeneous fluids $p_{0}$ is related 
to the Helmholtz free energy $f_{0}(\mathbf{n})$ in the following way:

$$
\begin{aligned}
p_{0} & =p_{0}(\mathbf{n}, T)=-\left(\frac{\partial F_{0}(\mathbf{n}, T, \Omega)}{\partial V}\right)_{T, \mathbf{N}}=-\left(\frac{\partial\left(f_{0}\left(\frac{\mathbf{N}}{V}, T\right) V\right)}{\partial V}\right)_{T, \mathbf{N}} \\
& =-f_{0}-V \sum_{i=1}^{M}\left(\frac{\partial f_{0}}{\partial n_{i}}\right)_{T, n_{1}, \ldots, n_{i-1}, n_{i+1}, \ldots, n_{M}}\left(\frac{\partial \frac{N_{i}}{V}}{\partial V}\right)_{N_{i}}-f_{0} \\
& =\sum_{i=1}^{M} n_{i}\left(\frac{\partial f_{0}}{\partial n_{i}}\right)_{T, n_{1}, \ldots, n_{i-1}, n_{i+1}, \ldots, n_{M}}- \\
& =\sum_{i=1}^{M} n_{i} \mu_{0, i}-f_{0} .
\end{aligned}
$$

Substituting the Peng-Robinson express of the Helmholtz free energy (2.6) into the above equation, we have

$$
p_{0}=\frac{n R T}{1-b n}-\frac{n^{2} a(T)}{1+2 b n-b^{2} n^{2}}=\frac{R T}{v-b}-\frac{a(T)}{v(v+b)+b(v-b)} .
$$

If one wants to solve for the molar volume $v=1 / n$ for a given pressure, the equation can be rearranged into an equation involving a cubic algebraic function of $v$, which is why this model is call a "cubic" EOS.

2.4. Influence parameter for modeling interface properties. The phase interface is modeled by the influence parameters $c_{i j}$. Like the parameters in the PengRobinson EOS, the influence parameter for a fluid mixture can be also related to the parameter of pure substance by a certain mixing rule. Here we adopt the modified geometric mean as the mixing rule:

$$
c_{i j}=\left(1-\beta_{i j}\right) \sqrt{c_{i} c_{j}},
$$

where the parameter $\beta_{i j}$ is the binary interaction coefficient for the influence parameter. Stability of the interface requires $\beta_{i j}$ to be included between 0 and 1 and $\beta_{i j}=\beta_{j i}$. For most systems, $\beta_{i j}$ is assumed to be zero. When $\beta_{i j}=0$, the mixing rule is reduced to the simple geometric mean.

The influence parameter of pure substance $c_{i}$ is related to the Peng-Robinson $a_{i}$ and $b_{i}$ by $[2,17,18]$

$$
c_{i}=a_{i} b_{i}^{2 / 3}\left(m_{1, i}^{c}\left(1-\frac{T}{T_{c_{i}}}\right)+m_{2, i}^{c}\right)
$$

where $m_{1, i}^{c}$ and $m_{2, i}^{c}$ are the coefficients correlated merely with the acentric factor $\omega_{i}$ of the component $i$ by the following relations:

$$
m_{1, i}^{c}=-\frac{10^{-16}}{1.2326+1.3757 \omega_{i}}, m_{2, i}^{c}=\frac{10^{-16}}{0.9051+1.5410 \omega_{i}} .
$$

3. Numerical solution strategies through time-dependent PDEs. In this paper, we are interested in the static behavior of two-phase fluid systems, such as the steady state profiles of molar density and stress. In other words, we would like to find a solution to the chemical equilibrium equation (2.5), or equivalently a solution 
to the original minimization problem of total Helmholtz free energy. Even though the numerical solution schemes presented here can be extended to multicomponent two-phase systems, for convenience of presentation, we restrict ourselves to singlecomponent two-phase systems (i.e., the fluid being pure substance). In this case, the chemical equilibrium equation (2.5) reduces to a scalar nonlinear second-order elliptic PDE:

$$
-c \Delta n=\mu-\mu_{0}(n) .
$$

The corresponding total Helmholtz free energy $F$ is

$$
F(n)=\int_{\Omega} f(n) d \mathbf{x}=\int_{\Omega}\left(f_{0}(n)+\frac{1}{2} c \nabla n \cdot \nabla n\right) d \mathbf{x} .
$$

The above PDE appears simple in its form, but due to the strong nonlinearity on the right-hand side of the PDE and the fact that the influence parameter $c$ is significantly small, its solution is computationally challenging. A straightforward approach is that we use a spatial discretization scheme (for example, a finite element method) to convert the PDE to a nonlinear algebraic equation system, and then we apply an iterative nonlinear solver (e.g. Newton's method) to obtain the solution of the algebraic equation system iteratively. This iterative numerical scheme for solving the time-independent PDE directly could be effective, provided that we overcome some of their challenges, such as stability issues in the nonlinear iteration. In certain cases, if the solution is not unique but the objective is to find one of the solutions, this approach to solving the time-independent elliptic PDE directly usually faces additional difficulties.

In this work, we pursue another approach, namely, first converting the above steady state problem into a time-dependent transient PDE whose solution at a sufficiently large time approaches our desired steady state solution. This approach, once combined with certain spatial and temporal discretization, can be used to provide the final steady state solution. In addition to the desired physical correspondence of the time-independent steady state solution, the transient PDE approach sometimes also has physically meaningful interpretation for its time-dependent solution, most of the time in a qualitative sense but sometimes even in a quantitative sense. In this paper, we focus on stable, efficient, and robust numerical discretization schemes for the transient PDE converted from the above static equilibrium equation.

3.1. Conversion to a second order time-dependent PDE. By adding a time-derivative term to the left-hand side of (2.5), this chemical equilibrium equation becomes

$$
\frac{\partial n(\mathbf{x}, t)}{\partial t}-c \Delta n(\mathbf{x}, t)=\mu-\mu_{0}(n(\mathbf{x}, t)), \quad \mathbf{x} \in \Omega \subset \mathbb{R}^{d} .
$$

With proper initial conditions and boundary conditions together with a specified bulk chemical potential $\mu$, the above PDE has a unique solution.

Even though the above PDE can be used to define a unique time-dependent solution, the solution is quite sensitive to the parameter $\mu$. In fact, our numerical testing indicates that a slight change of the bulk chemical potential $\mu$ causes a significant change of the solution. Another problem for the above second-order PDE formulation is that it is not mass conservative. The nonconservation is clear because of the 
nonzero right-hand source/sink term, which can cause either increase or decrease of the total mass in the system, even if we impose the homogeneous Neumann boundary condition. For most realistic cases, this lack of conservation will lead to the final steady state solution being either single gas phase (due to decrease of the mass) or single liquid phase (due to increase of the mass), a result of no interest to us.

3.1.1. Mass conservation. To fix these two problems, we incorporate a Lagrange multiplier to enforce mass conservation. That is, we treat $\mu$ as a unknown parameter that is constant spatially but could change with time. This allows us to impose an additional equation for enforcing mass conversation at all times:

$$
\begin{aligned}
\frac{\partial n(\mathbf{x}, t)}{\partial t}-c \Delta n(\mathbf{x}, t) & =\mu(t)-\mu_{0}(n(\mathbf{x}, t)), \quad \mathbf{x} \in \Omega \subset \mathbb{R}^{d} \\
\int_{\Omega} n(\mathbf{x}, t) d \mathbf{x} & =N \\
n(\mathbf{x}, t) & =n_{B}(\mathbf{x}, t), \quad \mathbf{x} \in \partial \Omega \\
n(\mathbf{x}, t) & =n_{\text {init }}(\mathbf{x}), \quad t=0
\end{aligned}
$$

Here we have closed the system by providing a standard initial condition and a Dirichlet boundary condition. The initial condition can be used to specify the total mass of the system (which is conservative through the time evolution) and consequently specify the ratio of liquid and gas amount. Besides Dirichlet boundary conditions, various boundary conditions can be implemented for different physical processes. Here we adopt the Dirichlet boundary condition to describe wettability of solid surfaces, which include, for example, fully liquid-wetting solid surfaces or fully vapor-wetting solid surfaces. In practice, if we want to have all boundaries be completely gas wetting, we can then specify $n_{B}(\mathbf{x}, t)$ to be the molar density of the gas bulk phase.

It is easy to show that if the solution $n(\mathbf{x}, t)$ approaches a steady state solution $n_{\text {steady }}(\mathbf{x})$ as the time $t$ goes to infinity, the chemical potential function $\mu(t)$ must also approach the desired chemical potential of bulk phase $\mu_{\text {steady }}$. We note that, however, $\mu(t)$ might differ from $\mu_{\text {steady }}$ before convergence in time.

3.1.2. Energy dissipation. In the following lemma, we can observe the dissipation of the total Helmholtz free energy with time.

Lemma 3.1 (energy identity). If $n(\mathbf{x}, t)$ is a solution of (3.2)-(3.5), then the following energy identity holds:

$$
\frac{d F(n)}{d t}+\left\|\frac{\partial n}{\partial t}\right\|_{L^{2}}^{2}=0
$$

Proof. We first recall the total Helmholtz free energy

$$
F(n)=\int_{\Omega} f(n) d \mathbf{x}=\int_{\Omega}\left(f_{0}(n)+\frac{1}{2} c \nabla n \cdot \nabla n\right) d \mathbf{x}
$$

Copyright (C) by SIAM. Unauthorized reproduction of this article is prohibited. 
where we have dropped temperature dependence due to the assumed isothermal condition. Taking a time derivative, we have

$$
\begin{aligned}
\frac{d F(n)}{d t} & =\int_{\Omega}\left(\frac{\partial f_{0}(n)}{\partial t}+c \nabla \frac{\partial n}{\partial t} \cdot \nabla n\right) d \mathbf{x} \\
& =\int_{\Omega} \frac{\partial n}{\partial t}\left(\mu_{0}-c \Delta n\right) d \mathbf{x}+\int_{\partial \Omega} c \frac{\partial n}{\partial t} \nabla n \cdot \nu d s \\
& =\int_{\Omega} \frac{\partial n}{\partial t}\left(\mu-\frac{\partial n}{\partial t}\right) d \mathbf{x}+\int_{\partial \Omega} c \frac{\partial n}{\partial t} \nabla n \cdot \nu d s \\
& =-\left\|\frac{\partial n}{\partial t}\right\|_{L^{2}}^{2} .
\end{aligned}
$$

We note that $\frac{\partial n}{\partial t}$ vanishes on the boundary due to the imposed time-independent Dirichlet boundary condition and that $\int_{\Omega} \frac{\partial n}{\partial t} \mu d \mathbf{x}$ vanishes because of the imposed mass conservation.

We remark that due to Lemma 3.1, the transient PDE approach can be viewed as a scheme to generate a sequence of molar density functions with decreased Helmholtz free energy and thus a scheme for the original minimization problem (2.2)-(2.3).

3.2. Energy stable semi-implicit time marching scheme through convex splitting of Peng-Robinson EOS. In this section, we discretize (3.2)-(3.5) by applying an energy stable time marching scheme. First of all, fully explicit time marching schemes for time integration do not work with our problem as expected. In fact, just to verify it, we implemented the fully explicit Euler's method, and numerical results (not shown here) indicate that the stability demands a time step to be really tiny so that the resultant computation is extremely time consuming. Moreover, somewhat surprising to us, fully implicit time marching schemes do not work as well. We also implemented the fully implicit Euler method, and numerical results (not shown here) again indicate a serious stability issue for implicit Euler (although not as bad as the issue for explicit Euler). To resolve this issue, a certain semi-implicit scheme is required for improved stability and for maintaining reasonable large time steps. We start our discussion by splitting the Peng-Robinson EOS into the summation of two parts, one being convex and the other one being concave. This splitting will lead to an efficient, stable, and energy-dissipated semi-implicit time marching scheme, as we will see below.

3.2.1. Convex splitting of Peng-Robinson EOS. In this analysis, we focus only on single-component systems, even though some of the conclusions (not all of them!) can extend to multicomponent systems. We note that the molar density must have values within the open interval $(0,1 / b)$ because of obvious physical constraints.

We first observe that the Helmholtz free energy density of homogeneous ideal gas is a convex function of molar density. This is very physically intuitive, because otherwise the fluid (with concave Helmholtz free energy density) would split into multiple phases!

Lemma 3.2. Helmholtz free energy density of homogeneous ideal gas is a convex function of molar density. In other words, $\partial^{2} f_{0}^{\text {ideal }}(n) / \partial n^{2}>0$.

Proof. We recall $f_{0}^{\text {ideal }}(n)=R T n(\ln n-1)$, which yields $\mu_{0}^{\text {ideal }}(n)=R T \ln n$, and $\frac{\partial^{2} f_{0}^{\text {ideal }}(n)}{\partial n^{2}}=\frac{\partial \mu_{0}^{\text {ideal }}(n)}{\partial n}=\frac{R T}{n}>0$.

Next we analyze the excess Helmholtz free energy density, which has two contributions, one from the repulsion or volume exclusion effect $\left(f_{0}^{b}\right)$ and the other from 
the molecular pairwise attraction $\left(f_{0}^{a}\right)$ :

$$
\begin{aligned}
f_{0}^{\text {excess }}(n) & =f_{0}^{a}(n)+f_{0}^{b}(n) \\
& =\frac{a(T) n}{2 \sqrt{2} b} \ln \left(\frac{1+(1-\sqrt{2}) b n}{1+(1+\sqrt{2}) b n}\right)-n R T \ln (1-b n) .
\end{aligned}
$$

Physically, the repulsion force must result in a convex contribution for the excess Helmholtz free energy density. This is clear at least when $n b$ approaches to 1 , where the Helmholtz free energy density approaches infinity. A rigorous mathematical statement is given as follows.

Lemma 3.3. The repulsion contribution of excess Helmholtz free energy density of homogeneous Peng-Robinson fluid is a convex function of molar density. In other words, $\partial^{2} f_{0}^{b}(n) / \partial n^{2}>0$.

Proof. A straightforward derivation yields $\mu_{0}^{b}(n)=\frac{\partial f_{0}^{b}(n)}{\partial n}=-R T \ln (1-b n)+$ $R T \frac{b n}{1-b n}$, and $\frac{\partial^{2} f_{0}^{b}(n)}{\partial n^{2}}=\frac{\partial \mu_{0}^{b}(n)}{\partial n}=\frac{b R T(2-b n)}{(1-b n)^{2}}>0$.

The attraction force must result in a nonconvex contribution for the excess Helmholtz free energy density, because otherwise phase separation will not occur. In fact, a quick mathematical derivation shows that it is strictly concave.

Lemma 3.4. The attraction contribution of excess Helmholtz free energy density of homogeneous Peng-Robinson fluid is a concave function of molar density. In other words, $\partial^{2} f_{0}^{a}(n) / \partial n^{2}<0$.

Proof. It is easy to see that

$$
\begin{aligned}
& \mu_{0}^{a}(n)=\frac{\partial f_{0}^{a}(n)}{\partial n}=\frac{a(T)}{2 \sqrt{2} b} \ln \left(\frac{1+(1-\sqrt{2}) b n}{1+(1+\sqrt{2}) b n}\right)-\frac{a(T) n}{1+b n+b n(1-b n)}, \\
& \frac{\partial^{2} f_{0}^{a}(n)}{\partial n^{2}}=\frac{\partial \mu_{0}^{a}(n)}{\partial n}=-\frac{a(T)}{1+b n+b n(1-b n)}-\frac{a(T)\left(1-b^{2} n^{2}\right)}{(1+b n+b n(1-b n))^{2}}<0 .
\end{aligned}
$$

The above analysis indicates that there exists a natural splitting of the PengRobinson EOS into summation of two terms:

$$
f_{0}(n)=\left(f_{0}^{\text {ideal }}(n)+f_{0}^{b}(n)\right)+f_{0}^{a}(n),
$$

where the first term $\left(f_{0}^{\text {ideal }}+f_{0}^{b}\right)$ is convex and the second term $f_{0}^{a}$ is concave. Similarly, the chemical potential from the Peng-Robinson EOS can be also split accordingly:

$$
\mu_{0}(n)=\left(\mu_{0}^{\text {ideal }}(n)+\mu_{0}^{b}(n)\right)+\mu_{0}^{a}(n) .
$$

3.2.2. Energy stability of a convex splitting scheme. We propose a semiimplicit, semidiscrete convex splitting scheme

$$
\begin{aligned}
\frac{n^{k+1}-n^{k}}{\Delta t}-c \Delta n^{k+1} & =\mu^{k+1}-\left(\mu_{0}^{\text {ideal }}\left(n^{k+1}\right)+\mu_{0}^{b}\left(n^{k+1}\right)\right)-\mu_{0}^{a}\left(n^{k}\right), \\
\int_{\Omega} n^{k+1} d \mathbf{x} & =N \\
n^{k+1}(\mathbf{x}) & =n_{B}^{k+1}, \quad \mathbf{x} \in \partial \Omega \\
n^{0}(\mathbf{x}) & =n_{\text {init }}(\mathbf{x}) .
\end{aligned}
$$

Copyright $@$ ㅇ by SIAM. Unauthorized reproduction of this article is prohibited. 
THEOREM 3.5. The semidiscrete scheme (3.6)-(3.9) is unconditionally energy stable, meaning that for any time step size $\Delta t>0$,

$$
F\left(n^{k+1}\right) \leq F\left(n^{k}\right) .
$$

Proof. Taking the $L^{2}$ inner product of (3.5) with $-\left(n^{k+1}-n^{k}\right)$ yields

$$
\begin{aligned}
-\frac{1}{\Delta t}\left\|n^{k+1}-n^{k}\right\|^{2}= & -\left(c \Delta n^{k+1}, n^{k+1}-n^{k}\right)-\left(\mu^{k+1}, n^{k+1}-n^{k}\right) \\
& +\left(\mu_{0}^{\text {ideal }}\left(n^{k+1}\right)+\mu_{0}^{b}\left(n^{k+1}\right), n^{k+1}-n^{k}\right)+\left(\mu_{0}^{a}\left(n^{k}\right), n^{k+1}-n^{k}\right) .
\end{aligned}
$$

From (3.7), $\int_{\Omega} \mu^{k+1} n^{k+1} d \mathbf{x}=\int_{\Omega} \mu^{k+1} n^{k} d \mathbf{x}$, and together with (3.8) we have

$$
\begin{aligned}
-\frac{1}{\Delta t}\left\|n^{k+1}-n^{k}\right\|^{2}= & c\left(\nabla n^{k+1}, \nabla n^{k+1}-\nabla n^{k}\right)+\left(\left.\frac{\partial f_{0}^{\text {ideal }}}{\partial n}\right|_{n=n^{k+1}}, n^{k+1}-n^{k}\right) \\
& +\left(\left.\frac{\partial f_{0}^{b}}{\partial n}\right|_{n=n^{k+1}}, n^{k+1}-n^{k}\right)+\left(\left.\frac{\partial f_{0}^{a}}{\partial n}\right|_{n=n^{k}}, n^{k+1}-n^{k}\right) .
\end{aligned}
$$

Utilizing the inequality $p(p-q) \geq \frac{1}{2}\left(p^{2}-q^{2}\right)$, we have

$$
c\left(\nabla n^{k+1}, \nabla n^{k+1}-\nabla n^{k}\right) \geq \frac{c\left\|n^{k+1}\right\|^{2}}{2}-\frac{c\left\|n^{k}\right\|^{2}}{2} .
$$

According to Lemma $3.2, f_{0}^{\text {ideal }}(n)$ is convex with respect to $n$, and thus

$$
\left.\frac{\partial f_{0}^{\text {ideal }}}{\partial n}\right|_{n=n^{k+1}}\left(n^{k+1}-n^{k}\right) \geq f_{0}^{\text {ideal }}\left(n^{k+1}\right)-f_{0}^{\text {ideal }}\left(n^{k}\right) .
$$

Similarly, from Lemma 3.3,

$$
\left.\frac{\partial f_{0}^{b}}{\partial n}\right|_{n=n^{k+1}}\left(n^{k+1}-n^{k}\right) \geq f_{0}^{b}\left(n^{k+1}\right)-f_{0}^{b}\left(n^{k}\right),
$$

and from Lemma 3.4,

$$
\left.\frac{\partial f_{0}^{a}}{\partial n}\right|_{n=n^{k}}\left(n^{k+1}-n^{k}\right) \geq f_{0}^{a}\left(n^{k+1}\right)-f_{0}^{a}\left(n^{k}\right) .
$$

From (3.11) and (3.12)-(3.15), we have

$$
\begin{aligned}
-\frac{1}{\Delta t}\left\|n^{k+1}-n^{k}\right\|^{2} & \geq \frac{c\left\|\nabla n^{k+1}\right\|^{2}}{2}-\frac{c\left\|\nabla n^{k}\right\|^{2}}{2}+\int_{\Omega} f_{0}\left(n^{k+1}\right) d \mathbf{x}-\int_{\Omega} f_{0}\left(n^{k}\right) d \mathbf{x} \\
& =F\left(n^{k+1}\right)-F\left(n^{k}\right) .
\end{aligned}
$$

Thus, $F\left(n^{k+1}\right) \leq F\left(n^{k}\right)$.

3.3. Full discretization by a conservative finite element method. In this work, we solve (3.2)-(3.5) using the Raviart-Thomas mixed finite element method, which is well known for its local mass conservation for approximate solutions, a property we desire to have here. This approach has been used frequently in reservoir engineering practice. For simplicity, we consider only a two- or three-dimensional rectangular domain $\Omega=\prod_{i=1}^{d}\left(0, L_{i}\right), d=2$ or 3 , and we consider only rectangular meshes. However, the results can be directly extended to a logically rectangular

Copyright $@$ by SIAM. Unauthorized reproduction of this article is prohibited. 
domain/mesh by conforming mapping. Let $\mathcal{T}_{h>0}$ be a quasi-uniform family of rectangular partitions of $\Omega$. The set of all interior edges (for a two-dimensional domain) or faces (for a three-dimensional domain) for $\mathcal{T}_{h}$ are denoted by $\Gamma_{h}$. On each edge or face $e \in \Gamma_{h}$, a unit normal vector $\nu_{e}$ is arbitrarily fixed. On the domain boundary, the normal vector $\nu_{e}$ coincides with the outward unit normal vector. We define

$$
\begin{aligned}
\mathbf{V} & \equiv H(\Omega ; \operatorname{div}) \equiv\left\{\mathbf{u} \in\left(L^{2}(\Omega)\right)^{d}: \operatorname{div} \mathbf{u} \in L^{2}(\Omega)\right\}, \\
W & \equiv L^{2}(\Omega) .
\end{aligned}
$$

The inner product in $\left(L^{2}(\Omega)\right)^{d}$ or $L^{2}(\Omega)$ is indicated by $(\cdot, \cdot)$, and the inner product in boundary function space $L^{2}(\Gamma)$ is indicated by $(\cdot, \cdot)_{\Gamma}$.

We first rewrite (3.2)-(3.5) in a weak formulation: find solution $n \in W$ and $\mathbf{u} \in \mathbf{V}$ such that the following equations hold:

$$
\begin{aligned}
\left(\frac{\partial n}{\partial t}, w\right)= & (\mathbf{u}, \nabla w)-\sum_{E \in \mathcal{T}_{h}}\left(\mathbf{u} \cdot \nu_{\partial E}, w\right)_{\partial E} \\
& +\left(\mu(t)-\mu_{0}(n), w\right) \quad \forall w \in W, \\
(1 / c)(\mathbf{u}, \mathbf{v})= & (n, \nabla \cdot \mathbf{v})-\left(n_{B}, \mathbf{v} \cdot \nu_{\partial \Omega}\right)_{\partial \Omega} \quad \forall \mathbf{v} \in \mathbf{V}, \\
\int_{\Omega} n d \mathbf{x}= & N, \\
(n, w)= & \left(n_{\text {init }}, w\right), \quad t=0 \quad \forall w \in W .
\end{aligned}
$$

Let the approximating subspace $\mathbf{V}_{s}\left(\mathcal{T}_{h}\right) \times W_{s}\left(\mathcal{T}_{h}\right)$ of $V \times W$ be the $s$ th-order $(s \geq 0)$ Raviart-Thomas space $\left(R T_{s}\right)$ of the partition $\mathcal{T}_{h}$. For example, for twodimensional domain $\Omega$, it is defined as

$$
\begin{aligned}
\mathbf{V}_{s}\left(\mathcal{T}_{h}\right) & =\left\{\mathbf{v} \in H(\Omega ; \operatorname{div}):\left.\mathbf{v}\right|_{R} \in Q_{s+1, s}(R) \times Q_{s, s+1}(R), R \in \mathcal{T}_{h}\right\}, \\
W_{s}\left(\mathcal{T}_{h}\right) & =\left\{w \in L^{2}(\Omega):\left.w\right|_{R} \in Q_{s, s}(R), R \in \mathcal{T}_{h}\right\},
\end{aligned}
$$

where we denote by $Q_{i, j}(R)$ the space of polynomials of degree less than or equal to $i$ (or $j$ ) in the first (or second) variable restricted to $R$. In the examples presented in this paper, we apply the lowest-order Raviart-Thomas space $\left(\mathrm{RT}_{0}\right)$. Its degrees of freedom for each rectangular element is five (four for flux variables on the four sides and one for the molar density at the center of the element).

The mixed finite element approximation to (3.2)-(3.5) can be formulated as follows: find $n_{h} \in W_{s}\left(\mathcal{T}_{h}\right)$ and $\mathbf{u}_{h} \in \mathbf{V}_{s}\left(\mathcal{T}_{h}\right)$ such that the following equations hold:

$$
\begin{aligned}
\sum_{E \in \mathcal{T}_{h}}\left(\frac{\partial n_{h}}{\partial t}, w_{h}\right)_{E}= & \sum_{E \in \mathcal{T}_{h}}\left(\mathbf{u}_{h}, \nabla w_{h}\right)_{E}-\sum_{E \in \mathcal{T}_{h}}\left(\mathbf{u}_{h} \cdot \nu_{\partial E}, w_{h}\right)_{\partial E} \\
& +\sum_{E \in \mathcal{T}_{h}}\left(\mu(t)-\mu_{0}\left(n_{h}\right), w_{h}\right)_{E} \quad \forall w_{h} \in W_{s}\left(\mathcal{T}_{h}\right) \\
\sum_{E \in \mathcal{T}_{h}}(1 / c)\left(\mathbf{u}_{h}, \mathbf{v}_{h}\right)_{E}= & \sum_{E \in \mathcal{T}_{h}}\left(n_{h}, \nabla \cdot \mathbf{v}_{h}\right)_{E}-\left(n_{B}, \mathbf{v}_{h} \cdot \nu_{\partial \Omega}\right)_{\partial \Omega} \quad \forall \mathbf{v}_{h} \in \mathbf{V}_{s}\left(\mathcal{T}_{h}\right), \\
\sum_{E \in \mathcal{T}_{h}} \int_{E} n_{h} d \mathbf{x}= & N \\
\sum_{E \in \mathcal{T}_{h}}\left(n_{h}, w_{h}\right)_{E}= & \sum_{E \in \mathcal{T}_{h}}\left(n_{\text {init }}, w_{h}\right)_{E}, \quad t=0 \quad \forall w_{h} \in W_{s}\left(\mathcal{T}_{h}\right)
\end{aligned}
$$

Copyright (c) by SIAM. Unauthorized reproduction of this article is prohibited. 
TABLE 1

Relevant data of isobutane $\left(\mathrm{nC}_{4}\right)$ (excerpted from Table 3.1 on page 141 of [8]).

\begin{tabular}{|c|c|c|c|c|}
\hline Component & Symbol & $T_{c}, \mathrm{~K}$ & $P_{c}, \mathrm{MPa}$ & $T_{b}, \mathrm{~K}$ \\
\hline \hline Isobutane & $n \mathrm{C}_{4}$ & 425.18 & 3.797 & 272.64 \\
\hline
\end{tabular}

For lowest-order Raviart-Thomas space $\left(\mathrm{RT}_{0}\right)$, we can also apply the trapezoid quadrature rule for the first term in (3.19), i.e., $\left(\mathbf{u}_{h}, \mathbf{v}\right)$, to decouple the system and to get an explicit formula for each individual diffusive flux.

We now present the convex-splitting semi-implicit method to further discretize the mixed finite element formulation (3.18)-(3.21) in time. The fully discretized formulation reads as follows: find $n_{h}^{k+1} \in W_{s}\left(\mathcal{T}_{h}\right)$ and $\mathbf{u}_{h}^{k+1} \in \mathbf{V}_{s}\left(\mathcal{T}_{h}\right)$ such that the following equations hold:

$$
\begin{aligned}
\sum_{E \in \mathcal{T}_{h}}\left(\frac{n_{h}^{k+1}-n_{h}^{k}}{\Delta t}, w_{h}\right)_{E}= & \sum_{E \in \mathcal{T}_{h}}\left(\mathbf{u}_{h}^{k+1}, \nabla w_{h}\right)_{E}-\sum_{E \in \mathcal{T}_{h}}\left(\mathbf{u}_{h}^{k+1} \cdot \nu_{\partial E}, w_{h}\right)_{\partial E} \\
& +\sum_{E \in \mathcal{T}_{h}}\left(\mu^{k+1}-\mu_{0}^{\text {ideal }}\left(n_{h}^{k+1}\right)-\mu_{0}^{b}\left(n_{h}^{k+1}\right), w_{h}\right)_{E} \\
& -\sum_{E \in \mathcal{T}_{h}}\left(\mu_{0}^{a}\left(n_{h}^{k}\right), w_{h}\right)_{E} \quad \forall k \geq 0, \forall w_{h} \in W_{s}\left(\mathcal{T}_{h}\right), \\
\sum_{E \in \mathcal{T}_{h}}(1 / c)\left(\mathbf{u}_{h}^{k+1}, \mathbf{v}_{h}\right)_{E}= & \sum_{E \in \mathcal{T}_{h}}\left(n_{h}^{k+1}, \nabla \cdot \mathbf{v}\right)_{E}-\left(n_{B}, \mathbf{v} \cdot \nu_{\partial \Omega}\right)_{\partial \Omega} \\
& \forall k \geq 0, \forall \mathbf{v}_{h} \in \mathbf{V}_{s}\left(\mathcal{T}_{h}\right), \\
\sum_{E \in \mathcal{T}_{h}} \int_{E} n_{h}^{k+1} d \mathbf{x}= & N \quad \forall k \geq 0, \\
\sum_{E \in \mathcal{T}_{h}}\left(n_{h}^{0}, w_{h}\right)_{E}= & \sum_{E \in \mathcal{T}_{h}}\left(n_{\text {init }}, w_{h}\right)_{E} \quad \forall w_{h} \in W_{s}\left(\mathcal{T}_{h}\right) .
\end{aligned}
$$

Proposition 3.6. The semi-implicit fully discrete scheme (3.22)-(3.25) is unconditionally energy stable. More precisely, for any time step $\Delta t>0$, there holds that

$$
F_{h}\left(n_{h}^{k+1}\right) \leq F_{h}\left(n_{h}^{k}\right)
$$

Here the discrete energy $F_{h}$ corresponding to the analytical energy functional (3.1) is defined as

$$
F_{h}\left(n_{h}\right)=\sum_{E \in \mathcal{T}_{h}} \int_{E}\left(f_{0}\left(n_{h}\right)+\frac{c}{2}\left|\nabla_{h} n_{h}\right|^{2}\right) d \mathbf{x}
$$

Proof. The detailed proof of Theorem 3.5 can be directly extended to the fully discrete schemes (3.22)-(3.25) and the discrete energy (3.26). Finally, the following inequality holds: $F_{h}\left(n_{h}^{k+1}\right)-F_{h}\left(n_{h}^{k}\right) \leq-\frac{1}{\Delta t} \sum_{E \in \mathcal{T}_{h}} \int_{E}\left(n_{h}^{k+1}-n_{h}^{k}\right)^{2} d \mathbf{x} \leq 0$.

4. Numerical experiments. In the numerical example below, we consider the species of isobutane $\left(n \mathrm{C}_{4}\right)$ at the temperature of $350 \mathrm{~K}$. The critical properties and the normal boiling point of this species are provided in Table 1.

We consider the problem of (3.2)-(3.5) on a two-dimensional domain $\Omega=(0, L)^{2}$, where $L=2 \times 10^{-8}$ meters. The initial condition is to impose the liquid density of 
isobutane under a saturated pressure condition at $350 \mathrm{~K}$ in the region of $(0.3 L, 0.7 L)^{2}$, and the rest of the domain is filled with a saturated gas of isobutane under the same temperature. In other words, $16 \%$ of the total volume is saturated liquid, while the rest (84\%) is saturated gas. The boundary condition is chosen to enforce the complete gas wetting condition on the entire boundary of the domain. The spatial discretization is carried out on the uniform $200 \times 200$ rectangular mesh. The time scale does not correspond to any physical process. We are only interested in the static behavior of the system. And since our time marching scheme is unconditionally stable, we take a relatively large time step size $\Delta t=10^{10}$ seconds for all time steps.

4.1. Spatial distributions of molar density and other chemical properties. Initially there is a jump in molar density between the gas and liquid regions, which gives a large (infinity in fact) gradient contribution to total Helmholtz free energy. After only one time step, a finite-thickness gas-liquid interface is formed (not shown); however the shape of the droplet is still close to the square shape imposed initially. After 10 time steps, the droplet shape is square but with four corners being rounded; see Figure 2(a) for the corresponding distribution of molar density, where the red color (discernible only in the online version) illustrates the liquid droplet. After 30 time steps, the droplet becomes a circular-like shape (see Figure 2(e)) and the droplet shape in the converged solution appears to be a perfect circle (see Figure $3(\mathrm{a}))$ as expected.

To indicate the interface itself clearly, we define the surface tension contribution of Helmholtz free energy density as

$$
f_{\text {intfTens }}=2 f_{\nabla}(n)=c \nabla n \cdot \nabla n .
$$

It is easy to see that

$$
\begin{aligned}
& F(n)-F_{0}\left(n_{\text {init }}\right) \\
= & \int_{\Omega}\left(f_{0}(n)-f_{0}\left(n_{\text {init }}\right)\right) d \mathbf{x}+\int_{\Omega} f_{\nabla}(n) d \mathbf{x} \cong \int_{\Omega} f_{\text {intfTens }} d \mathbf{x} .
\end{aligned}
$$

Figures 2(b), (d), (f) and Figure 3(b) display the surface tension contribution of Helmholtz free energy density at different time steps, where the red color (discernible only in the online version) illustrates the gas-liquid interface.

The total chemical potential $\mu$ must be a constant across the entire domain, but the homogeneous contribution of chemical potential $\mu_{0}$ can vary spatially, as shown in Figure $3(\mathrm{c})$. In particular, $\mu_{0}$ changes dramatically across the interface even though it must have the same value in the liquid and gas bulk regions.

The strict dissipation of the total Helmholtz free energy with time steps is clear in Figure 3(e). As expected, Figure 3(e) demonstrates that the energy decay is fast initially but quickly slows down at later time steps when the solution approaches its steady state. Figure 3(f) is a zoom-in plot of Figure 3(e) in later time steps, illustrating that the total Helmholtz free energy remains to decrease all the time.

4.2. Calculation of interface tension and verification against YoungLaplace equation. The interface tension $\sigma$ is the quantity of interest in many applications. Mechanically, the surface tension is defined as the net contractive force per unit length of interface, with a unit of $\mathrm{N} / \mathrm{m}$. Thermodynamically, it is defined as the work for creating a unit area of interface, with a unit of $\mathrm{J} / \mathrm{m}^{2}$. Using the first law of thermodynamics, surface tension can be shown to relate to the Helmholtz energy by $\sigma=\frac{\partial F}{\partial A}$, where $A$ is the area of the interface from the sharp interface point of view. If 


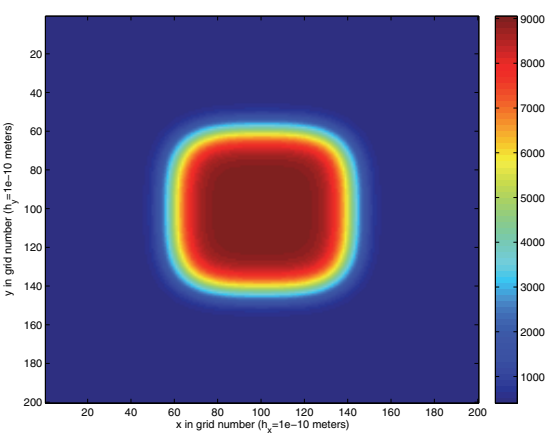

(a)

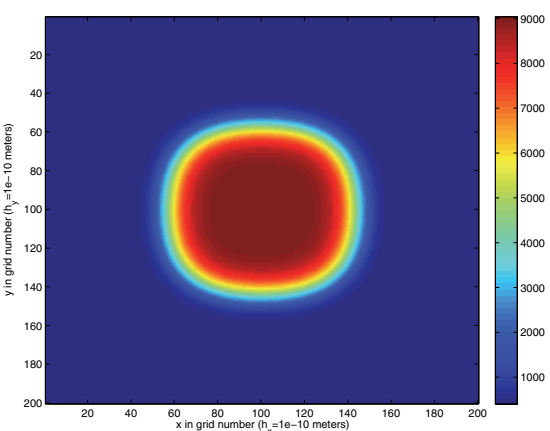

(c)

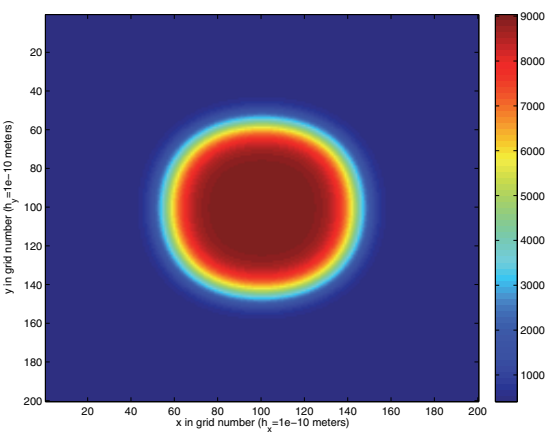

(e)

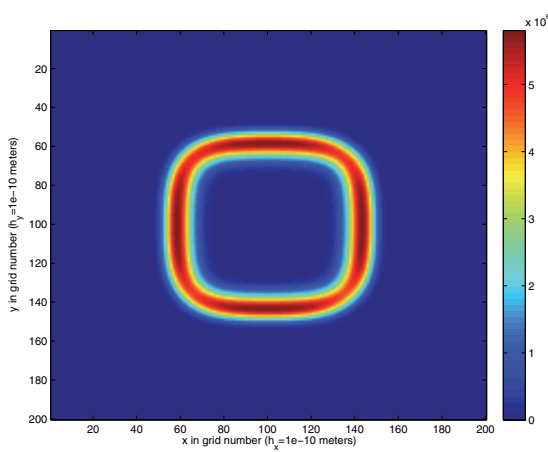

(b)

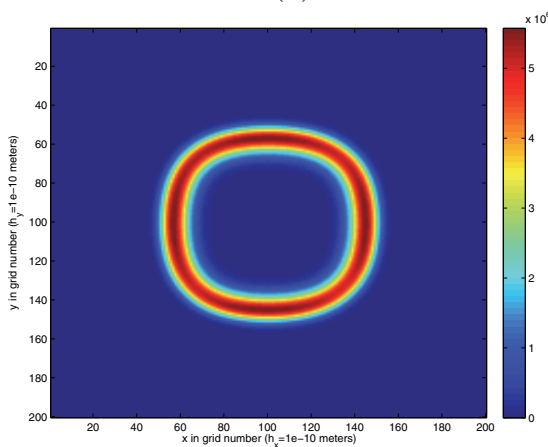

(d)

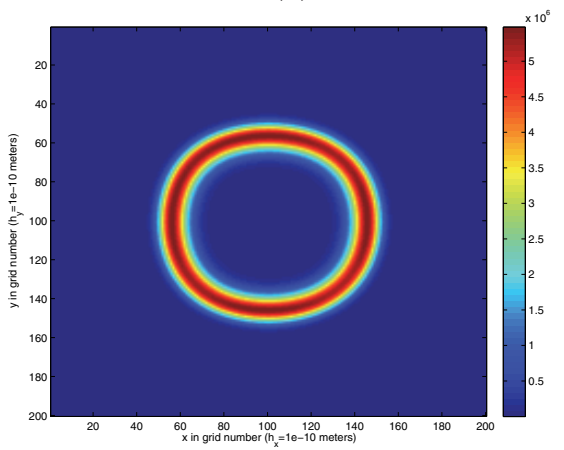

(f)

FIG. 2. Numerical results: (a) molar density (in mol/m^3) after 10 time steps; (b) surface tension contribution of Helmholtz free energy density (in $\mathrm{J} / \mathrm{m} \wedge$ ) after 10 time steps; (c) molar density (in mol/m $\mathrm{m}^{\wedge}$ ) after 20 time steps; (d) surface tension contribution of Helmholtz free energy density (in $\mathrm{J} / \mathrm{m}^{\wedge} 3$ ) after 20 time steps; (e) molar density (in mol/m^3) after 30 time steps; (f) surface tension contribution of Helmholtz free energy density (in $\mathrm{J} / \mathrm{m}^{\wedge} 3$ ) after 30 time steps.

we assume that surface tension $\sigma$ is spatially constant within the interface for a given system, we can compute the Helmholtz energy by $\sigma=\frac{F(n)-F_{0}\left(n_{\text {init }}\right)}{A}$.

In this example, we integrate the quality $f_{\text {intfTens }}$ over the entire domain to obtain $F(n)-F_{0}\left(n_{\text {init }}\right) \cong 1.763 \times 10^{-10} \mathrm{~J}$. Assuming that the volume of the droplet does not change with time and that the steady state droplet has a perfect circular shape, we can compute the radius of the droplet: $r=2 \times 10^{-8} \times(0.16 / \pi)^{1 / 2}=4.514 \times$ $10^{-9}$ meters. Then the surface tension is $\sigma=1.763 \times 10^{-10} /\left(2 \pi \times 4.514 \times 10^{-9}\right)=$ 


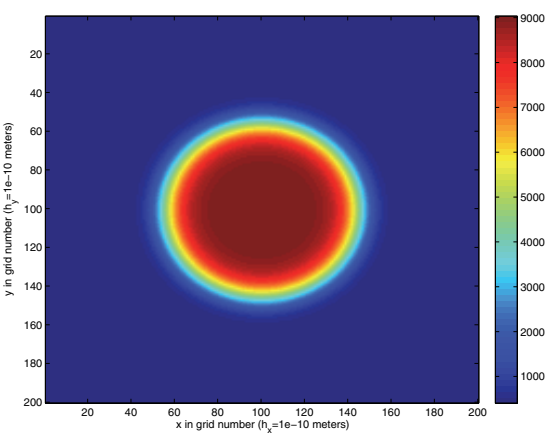

(a)

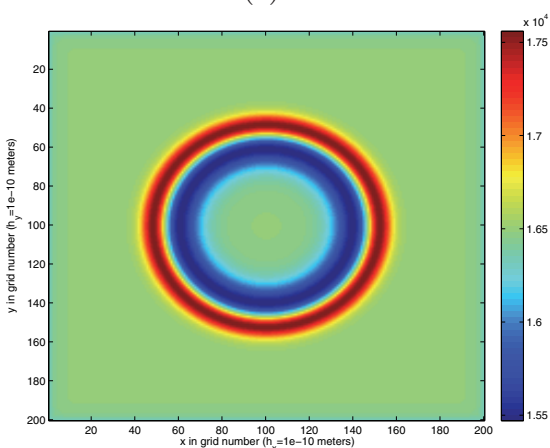

(c)

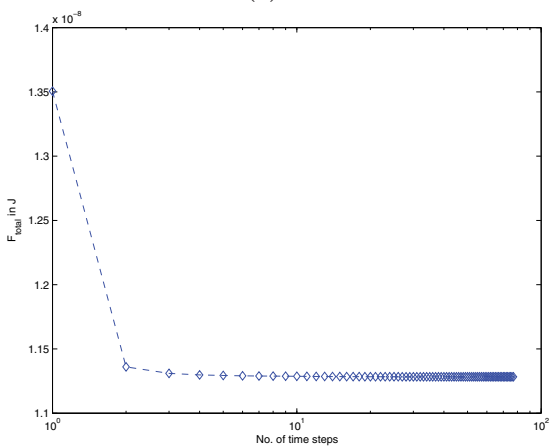

(e)

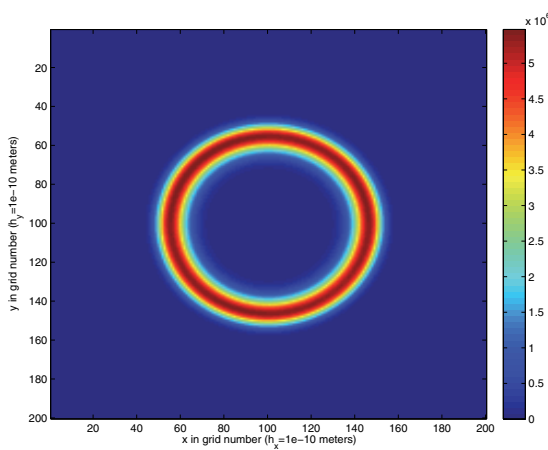

(b)

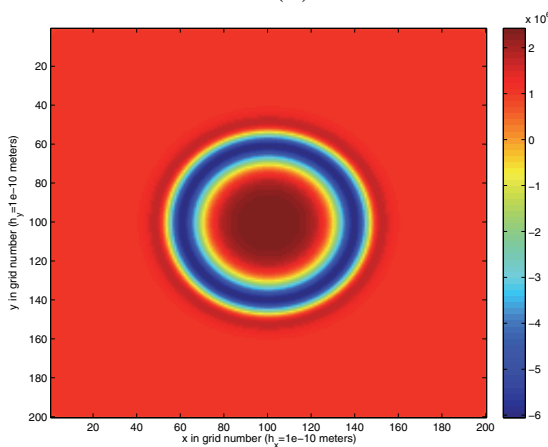

(d)

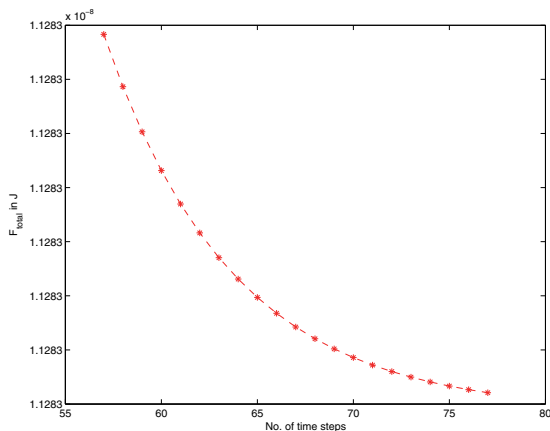

(f)

FIG. 3. Numerical results: (a) mole density (in mol $/ m^{\wedge} 3$ ) after convergence (77 time steps); (b) surface tension contribution of Helmholtz free energy density (in $\mathrm{J} / \mathrm{m}^{\wedge} 3$ ) after convergence (77 time steps); (c) homogeneous contribution of chemical potential (in $\mathrm{J} / \mathrm{mol}$ ) after convergence (77 time steps); (d) smallest component of stress tensor (in Pa) after convergence (77 time steps); (e) convergence history; (f) recent convergence history.

$6.216 \times 10^{-3} \mathrm{~J} / \mathrm{m}^{2}=6.216 \mathrm{mN} \cdot \mathrm{m}^{-1}$. We compute the surface tension of isobutane under the same setting except with various temperatures ranging from $250 \mathrm{~K}$ to $340 \mathrm{~K}$, and the results are plotted in Figure 4(a). In the same figure, the predicted values are compared with the laboratory measured values as reported in Table 2 of [16]. Clearly, the trend is captured and the difference between prediction and measurement (which come mainly from modeling errors) is small from an engineering point of view.

The well-known Young-Laplace equation can be written in the following form for 


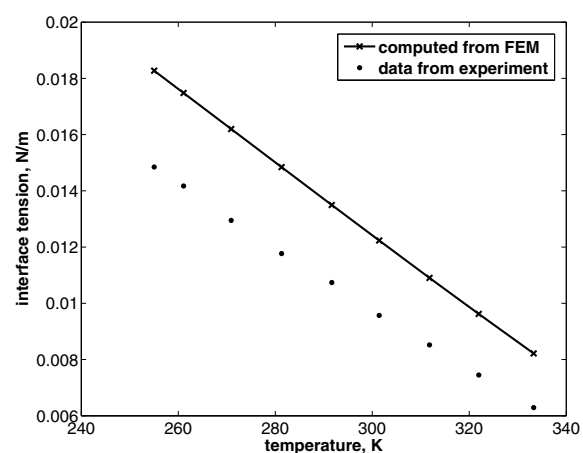

(a)

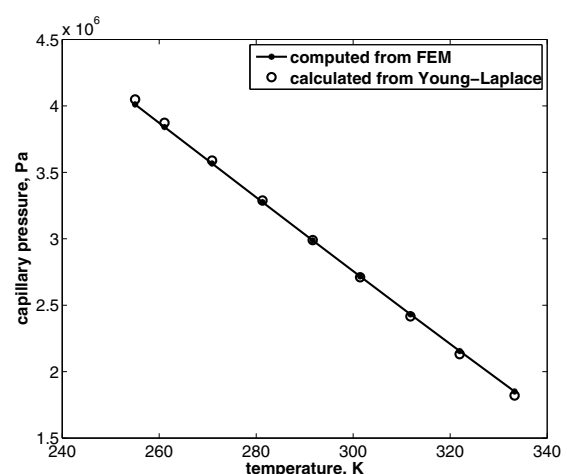

(b)

FIG. 4. Comparison between numerical predictions and laboratory data: (a) surface tension; (b) capillary pressure.

a droplet in two spatial dimensions [8]:

$$
P_{c}=P_{\text {liquid }}-P_{\text {gas }}=\frac{\sigma}{r} .
$$

In our numerical example, the pressure in the center of liquid drop is $P_{\text {liquid }}=2.4239 \times$ $10^{6} \mathrm{~Pa}$ and the pressure in the bulk gas region is $P_{\text {gas }}=1.0189 \times 10^{6} \mathrm{~Pa}$, with liquid pressure picked from element $(100,100)$ and gas pressure picked from element $(50,50)$. The difference between the two is the capillary pressure $P_{c}=1.405 \mathrm{MPa}$. On the other hand, the capillary pressure predicted by the Young-Laplace equation is $P_{c}=$ $6.216 \times 10^{-3} / 4.514 \times 10^{-9} \mathrm{~Pa}=1.377 \mathrm{MPa}$. Clearly, the Young-Laplace prediction is well matched with the capillary pressure observed in the finite element solution with less than $2 \%$ error. Capillary pressures predicted by the finite element simulation and those by the Young-Laplace equation are also plotted and compared for the same setting except with difference temperatures ranging from $250 \mathrm{~K}$ to $340 \mathrm{~K}$ (see Figure 4(b)). Agreement with errors around or smaller than $2 \%$, is observed under various temperatures consistently. This small error might come from two sources; one is the finite element discretization error, and the other is that the Young-Laplace equation is derived using sharp interface theory, which may not be exact in the context of diffuse interface theory.

We note that within the interface the stress tensor is anisotropic, where we plot its smallest component in Figure 3(d), while in the bulk region of both phases, the stress tensor is isotropic, which yields the liquid and gas pressures above.

5. Conclusion. Mixture of immiscible and partially miscible fluids commonly occurs in daily life and engineering practice. In this paper, two-phase fluid systems are modeled and simulated using a diffusive interface model with the Peng-Robinson equation of state (EOS), a realistic thermodynamic model for hydrocarbon and other reservoir fluids. Conventional phase field simulations usually use the simple doublewell potential instead of a realistic EOS and thus can simulate only a qualitative behavior of the multiphase systems. One main objective of this paper is to describe quantitative (rather than qualitative) behaviors of two phases with targeted application to petroleum fluids. Our model is derived based on first principles from thermodynamics. The minimization problem of Helmholtz free energy is first formulated from the classical theory of canonical ensembles. This minimization formulation, through 
variational calculus, naturally yields a balance equation of chemical potential, which is a second-order elliptic partial differential equation (PDE) in molar density with a strongly nonlinear source term.

The focus of the paper is to study the static behavior of two-phase fluid systems through the elliptic-type PDE that describes the chemical equilibrium condition; however, we solve the time-dependent PDE even though the final goal is to find its final steady state solution. The resultant second-order diffusion-type PDE is not mass conservative, and nonphysical solution drift often occurs during time stepping, which leads to undesired single-phase profiles. To avoid this difficulty, a Lagrange multiplier is used to enforce mass conservation. Our simulation approach is capable of predicting quantitatively the interface details between the two phases, which include the thickness of the interface and the magnitude of interface tension. In multiple dimensional simulation, it also predicts the shape of bubbles or droplets during various equilibrium conditions, including gravity. Among many renowned challenges, the considered problem has strong nonlinearity and high stiffness from the Peng-Robinson EOS. An effective semi-implicit time marching scheme is proposed, for the first time solving successfully the spatially heterogeneous two-phase systems with the Peng-Robinson EOS in multiple spatial dimensions. Our semi-implicit time scheme treats the volume exclusion effect of an EOS implicitly, while the pairwise attraction effect of an EOS is calculated explicitly. Our splitting not only has clear motivation from physics, but it is also shown to be a convex splitting and thus to yield an energy stable time marching scheme. Our proposed scheme is both mass conservative and energy dissipated, which is proved with mathematical rigor and is also verified with numerical experiments in the paper.

Finally, we provide a numerical example to illustrate the details within the interface. Our numerical example use a realistic hydrocarbon component (isobutane), and our prediction of surface tension matches well with experimental data. Our computational results are also verified with the well-known Young-Laplace equation.

An immediate future work is to extend the current implementation to treat multicomponent systems in three spatial dimensions. Additional future work of interest includes applications of the framework presented here to engineering problems arising in the petroleum industry.

Acknowledgment. We would like to thank the anonymous reviewers for their helpful suggestions.

\section{REFERENCES}

[1] K. BAO, Y. Shi, S. Sun, And X.P. WAng, A finite element method for the numerical solution of the coupled Cahn-Hilliard and Navier-Stokes system for moving contact line problems, J. Comput. Phys., 231 (2012), pp. 8083-8099.

[2] B. Breure And C.J. Peters, Modeling of the surface tension of pure components and mixtures using the density gradient theory combined with a theoretically derived influence parameter correlation, Fluid Phase Equil., 334 (2012), pp. 189-196.

[3] W. Chen, S. Conde, C. Wang, X. Wang, and S.M. Wise, A linear energy stable scheme for a thin film model without slope selection, J. Sci. Comput., 52 (2012), pp. 546-562.

[4] H.T. Davis, Statistical Mechanics of Phases, Interfaces, and Thin Films, VCH, New York, 1996.

[5] C. Dawson, S. Sun, And M.F. Wheeler, Compatible algorithms for coupled flow and transport, Comput. Methods Appl. Mech. Engrg., 193 (2004), pp. 2565-2580.

[6] Q. Du and R.A. Nicolaides, Numerical analysis of a continuum model of phase transition, SIAM J. Numer. Anal., 28 (1991), pp. 1310-1322.

[7] D.J. Eyre, An Unconditionally Stable One-Step Scheme for Gradient Systems, manuscript, 
http://www.math.utah.edu/ eyre/research/methods/stable.ps.

[8] A. Firoozabadi, Thermodynamics of Hydrocarbon Reservoirs, McGraw-Hill, New York, 1999.

[9] D. Frenkel and B. Smit, Understanding Molecular Simulation: From Algorithms to Applications, Academic Press, San Diego, CA, 2001.

[10] D. Furihata, A stable and conservative finite difference scheme for the Cahn-Hilliard equation, Numer. Math., 87 (2001), pp. 675-699.

[11] H. Gomez And T.J.R. Hughes, Provably unconditionally stable, second-order time-accurate, mixed variational methods for phase-field models, J. Comput. Phys., 230 (2011), pp. 53105327.

[12] F. Guillén-GonzÁlez And G. Tierra, On linear schemes for a Cahn-Hilliard diffuse interface model, J. Comput. Phys., 234 (2013), pp. 140-171.

[13] K.B. Haugen And A. Firoozabadi, Composition at the interface between multicomponent nonequilibrium fluid phases, J. Chem. Phys., 130 (2009), 064707.

[14] J. Kou AND S. Sun, An adaptive finite element method for simulating surface tension with the gradient theory of fluid interfaces, J. Comput. Appl. Math., 255 (2014), pp. 593-604.

[15] L.W. Lake, Enhanced Oil Recovery, Prentice-Hall, Englewood Cliffs, NJ, 1989.

[16] H. Lin AND Y.-Y. DuAn, Surface tension measurements of propane ( $r-290)$ and isobutane (r-600a) from (253 to 333) k, J. Chem. Eng. Data, 48 (2003), pp. 1360-1363.

[17] C. Miqueu, B. Mendiboure, A. Graciaa, and J. Lachaise, Modelling of the surface tension of pure components with the gradient theory of fluid interfaces: A simple and accurate expression for the influence parameters, Fluid Phase Equil., 207 (2003), pp. 225-246.

[18] C. Miqueu, B. Mendiboure, A. GraciaA, and J. Lachaise, Modeling of the surface tension of multicomponent mixtures with the gradient theory of fluid interfaces, Ind. Eng. Chem. Res., 44 (2005), pp. 3321-3329.

[19] J. Moortgat, S. Sun, And A. Firoozabadi, Compositional modeling of three-phase flow with gravity using higher-order finite element methods, Water Resour. Res., 47 (2011), W05511.

[20] L.C. Nielsen, I.C. Bourg, And G. Sposito, Predicting CO2-water interfacial tension under pressure and temperature conditions of geologic CO2 storage, Geochimica et Cosmochimica Acta, 81 (2012), pp. 28-38.

[21] A. Onuki, Phase Transition Dynamics, Cambridge University Press, Cambridge, UK, 2002.

[22] D.-Y. Peng and D.B. Robinson, A new two-constant equation of state, Ind. Eng. Chem. Fund., 15 (1976), pp. 59-64.

[23] Z. QIAO, Z. Zhang, AND T. TANG, An adaptive time-stepping strategy for the molecular beam epitaxy models, SIAM J. Sci. Comput., 33 (2011), pp. 1395-1414.

[24] L. Rongy, K.B. Haugen, and A. Firoozabadi, Mixing from Fickian diffusion and natural convection in binary non-equilibrium fluid phases, AIChE J., 58 (2012), pp. 1336-1345.

[25] J. Shen, C. WANG, X. WANG, AND S.M. Wise, Second-order convex splitting schemes for gradient flows with Ehrlich-Schwoebel type energy: Application to thin film epitaxy, SIAM J. Numer. Anal., 50 (2012), pp. 105-125.

[26] J. Shen And X. Yang, Numerical approximations of Allen-Cahn and Cahn-Hilliard equations, Discrete Contin. Dyn. Syst., 28 (2010), pp. 1669-1691.

[27] M. Struwe, Variational Methods: Applications to Nonlinear Partial Differential Equations and Hamiltonian Systems, Ergeb. Math. Grenzgeb. (3) 34, Springer, Berlin, 2008.

[28] S. Sun And J. Geiser, Multiscale discontinuous Galerkin and operator-splitting methods for modeling subsurface flow and transport, Int. J. Multiscale Comput. Engrg., 6 (2008), pp. 87-101.

[29] S. Sun And M.F. Wheeler, Symmetric and nonsymmetric discontinuous Galerkin methods for reactive transport in porous media, SIAM J. Numer. Anal., 43 (2005), pp. 195-219.

[30] S. Sun AND M.F. WheELeR, Local problem-based a posteriori error estimators for discontinuous Galerkin approximations of reactive transport, Comput. Geosci., 11 (2007), pp. 87-101.

[31] G. Tegze, T. Pusztai, G. Toth, L. Granasy, A. Svandal, T. Buanes, T. Kuznetsova, and B. Kvamme, Multiscale approach to CO2 hydrate formation in aqueous solution: Phase field theory and molecular dynamics. Nucleation and growth, J. Chem. Phys., 124 (2006), 234710.

[32] B.P. Vollmayr-Lee and A.D. Rutenberg, Fast and accurate coarsening simulation with an unconditionally stable time step, Phys. Rev. E (3), 68 (2003), 066703.

[33] C. WANG, X. WANG, AND S.M. WISE, Unconditionally stable schemes for equations of thin film epitaxy, Discrete Contin. Dyn. Syst., 28 (2010), pp. 405-423.

[34] C. Wang And S.M. Wise, An energy stable and convergent finite-difference scheme for the modified phase field crystal equation, SIAM J. Numer. Anal., 49 (2011), pp. 945-969.

[35] S.M. Wise, C. Wang, And J.S. Lowengrub, An energy-stable and convergent finite-difference scheme for the phase field crystal equation, SIAM J. Numer. Anal., 47 (2009), pp. 2269-

Copyright (c) by SIAM. Unauthorized reproduction of this article is prohibited. 
2288.

[36] C. Xu And T. TAng, Stability analysis of large time-stepping methods for epitaxial growth models, SIAM J. Numer. Anal., 44 (2006), pp. 1759-1779.

[37] Z. Zhang, Y. MA, AND Z. QIAO, An adaptive time-stepping strategy for solving the phase field crystal model, J. Comput. Phys., 249 (2013), pp. 204-215.

[38] Z. Zhang AND Z. QIAO, An adaptive time-stepping strategy for the Cahn-Hilliard equation, Commun. Comput. Phys., 11 (2012), pp. 1261-1278.

Copyright (c) by SIAM. Unauthorized reproduction of this article is prohibited. 